\title{
IndustryConnect: WI-Forschung mit und für die Praxis
}

\author{
Petra Schubert $\cdot$ Susan P. Williams
}

Eingegangen: 20. Oktober 2019 / Angenommen: 10. Februar 2020 / Online publiziert: 19. Februar 2020 (C) Der/die Autor(en) 2020

Zusammenfassung Der Beitrag beschreibt ein Rahmenwerk für praxisorientierte Langzeitforschung, das an Boyers vier Scholarships angelehnt ist. Das Modell wird im Rahmen der Initiative IndustryConnect seit mehr als fünf Jahren erfolgreich für die gemeinsame Forschung im Themengebiet Digitaler Arbeitsplatz eingesetzt. Die besonderen Erfolgsfaktoren der gemeinsamen Arbeit zwischen Wissenschaft und Praxis werden erläutert, wie zum Beispiel die Schaffung einer gemeinsamen Begriffswelt und das Anlegen einer gemeinsamen Wissensbasis. Das Rahmenwerk umfasst einen ausgewählten Methodenmix, inklusive der speziell entwickelten Methoden eXperience Fallstudien und Milestories, und der damit verbundenen Methodenausbildung für Doktoranden. Typische Ergebnisse aus dieser Art der Forschung sind Erklärungs- und Klassifikationsmodelle, Methoden und Prototypen, für die exemplarische Publikationen aufgeführt werden. Am Ende des Beitrags findet sich ein Bericht über Erfahrungen und Erkenntnisse aus der gemeinsamen Forschungsarbeit „mit und für die Praxis“ sowie eine Ermutigung, dem Aufruf von Nunamaker et al. zu folgen, die ,letzte Meile in der WI-Forschung zu gehen“.

Schlüsselwörter Wirtschaftsinformatik · Praxisorientierte Forschung · Fallstudien · Digitaler Arbeitsplatz · Kollaborationssysteme · CSCW 


\title{
IndustryConnect: IS Research with and for Practice
}

\begin{abstract}
In this article we describe a framework for long-term practice-oriented research with industry that is based on Boyer's four forms of scholarship. The framework has been used successfully for joint research on the implementation and adoption of the Digital Workplace for more than five years in the IndustryConnect Initiative. The particular success factors of the joint work between science and practice are explained, such as the creation of a common conceptual world and the development of a common knowledge base. The framework includes a set of mixed methods, including the specifically developed methods eXperience Case Studies and Milestories, and a tailored programme of research training for doctoral students. Typical results from this type of research are explanatory and classification models, methods and prototypes, for which exemplary publications are presented. The article concludes with a report on experiences and lessons learned from joint research work "with and for practitioners" as well as an endorsement of the call by Nunamaker et al. to go the "last mile in IS research".
\end{abstract}

Keywords Information Systems · University-Industry Collaboration · Case Studies · Digital Workplace · Enterprise Collaboration Systems · CSCW

\section{Methodendiskussion in der Wirtschaftsinformatik}

Die Forschung im Bereich Wirtschaftsinformatik und ihrer amerikanischen Schwesterdisziplin „Information Systems“ ist geprägt durch eine anhaltende Diskussion über die epistemologische Grundhaltung und die Methoden der wissenschaftlichen Forschung. Dazu erschienene Literaturbeiträge adressieren verschiedene Aspekte wie z.B. die wissenschaftstheoretische Grundhaltung (Schön 2001; Schneiderman 2016), Umfang und Relevanz des Untersuchungsobjekts (Lyytinen et al. 2007; Davison und Martinsons 2011; Winter und Butler 2011; Adler und Hansen 2012; Walsham 2012; Alvesson und Sandberg 2013) oder die eingesetzten Ansätze und Methoden (Davison und Martinsons 2011; Galliers 2011; Avison und Malaurent 2014). Galliers und Stein (2018) diskutieren gar die Frage, ob „Information Systems" überhaupt eine Wissenschaft ist.

Debates over the status of the IS field as a science (or not) and over its methods seem to do little in terms of helping the field produce exciting research and rather feed our collective anxieties about irrelevance (Galliers und Stein 2018, S. 12).

Der Methodenstreit kostet viel Energie, die der eigentlichen Forschung entgeht. Galliers und Stein (2018) nennen es „A Debate that Refuses to Die“. Einer der Punkte im anhaltenden Methodenstreit ist der Einbezug von Praxisvertretern in die Forschung, was in der Literatur als „University-Industry Collaboration“ diskutiert wird (Dooley und Kirk 2007; Schubert et al. 2015). Deutschland hat aufgrund seiner organisationalen Struktur (Lehrstühle und Forschergruppen) einen Vorteil und kann viele der diskutierten Probleme effektiv adressieren. Die Langfristigkeit von 
Professuren und das Zusammenbringen von jungen Forschern um ein gemeinsames Thema erlaubt die Gestaltung von Forschungsprogrammen statt nur kleinen Forschungsprojekten bzw. Publikationsprojekten. Die Sichtbarkeit und Langfristigkeit von Forschergruppen erleichtert darüber hinaus den Aufbau des notwendigen Vertrauens von Praktikern, die in die universitäre Forschung einbezogen werden können. Die gewählten Forschungsfragen werden von Praktikern mitgestaltet, die Ergebnisse basieren auf empirischen Daten, die mit rigorosen Methoden erhoben und ausgewertet werden und die Ergebnisse werden am Ende in die Praxis zurücktransferiert und dort evaluiert. Damit demonstriert ein Großteil der deutschen WI-Forschung das erfolgreiche Gehen der von Nunamaker et al. geforderten „Last Research Mile“ (Nunamaker et al. 2015).

Werfen wir einen genaueren Blick auf Deutschland. Dort wurde im März 2010, also vor knapp 10 Jahren, das „Memorandum zur gestaltungsorientierten Wirtschaftsinformatik" von einer Professorengruppe aus zehn WKWI-Mitgliedern veröffentlicht (Österle et al. 2010). In der Folge wurde das Memorandum in englischer Sprache im European Journal of Information Systems (Österle et al. 2011) der internationalen Forschungsgemeinschaft zugänglich gemacht, was zu einer interessanten Reaktion aus dem Lager der US-amerikanischen IS-Forscher führte (Baskerville et al. 2011). Schon die Überschrift des Artikels suggeriert, dass die Publikationstätigkeit die Maßeinheit für einflussreiche Forschungsergebnisse (,high-impact IS research“) ist.

Doch ist eine intensive Publikationstätigkeit wirklich das, was die Anspruchsgruppen von der Forschung an deutschen Hochschulen erwarten? Eine vermeintliche Antwort findet sich in der deutschen Gesetzgebung. Der Leistungsauftrag deutscher Hochschulen ist in den Hochschulgesetzen der jeweiligen Länder geregelt. Gemeinsam ist diesen Gesetzen der Auftrag der Forschung und der Lehre. Im Hochschulgesetz des Landes RLP (HochSchG, 19.11.2010) wird die Forschung beschrieben als „Gewinnung wissenschaftlicher Erkenntnisse“ und die Lehre als „Grundlegung und Weiterentwicklung von Lehre und Studium“. Andere Landesgesetze verankern zusätzliche Ziele. In NRW (HG, 16.09.2014) sind z.B. in § 3 zusätzlich explizit die Förderung des wissenschaftlichen Nachwuchses und der Wissenstransfer genannt. Das Hessische Gesetz (HHG, 19.11.2010) nennt darüber hinaus in $\S 3$ (3) explizit die Unterstützung der Absolventinnen und Absolventen bei der Existenzgründung. Der Auftrag eines (deutschen) Professors ist demnach vielfältig, aber glücklicherweise bietet die Wirtschaftsinformatik ideale Möglichkeiten, Forschung, Lehre, Nachwuchsförderung und Existenzgründung miteinander zu verbinden.

Dies bringt uns zum Fokusthema dieses Beitrags. Dieser Artikel ist ein reflexiver Bericht über den Auf- und Ausbau einer Forschungsinitiative (IndustryConnect), die Vertreter aus Wirtschaft und Wissenschaft über viele Jahre in einer gemeinsamen Untersuchung der Entwicklung des Digitalen Arbeitsplatzes in Unternehmen zusammengebracht hat. IndustryConnect ist eine Erfolgsgeschichte, die - wie viele ihrer Art - von glücklichen Zufällen (Serendipity), aber auch von gezielter Planung und Fleiß geschrieben wurde. Die Erfolgsfaktoren von IndustryConnect liegen in der Kombination rigoroser Forschungsmethoden in Verbindung mit einer vertrauensvollen Gemeinschaft von Forschenden und Praktikern. Sind die Faktoren von IndustryConnect übertragbar auf andere Forschungsinitiativen und ist die damit ver- 
bundene, praxisorientierte Forschung für die Forschenden lohnend? Der folgende Beitrag gibt eine Antwort auf diese Frage.

\section{Rahmenmodell praxisorientierter Forschung}

Ausgangspunkt von IndustryConnect ist ein gemeinsames Fokusthema, für das sich sowohl Wissenschaftler als auch Praktiker interessieren. Das dadurch entstandene, übergeordnete Forschungsprogramm fokussiert auf die Digitalisierung in Unternehmen, bzw. speziell auf die Gestaltung des Digitalen Arbeitsplatzes. Die Forschung in diesem Bereich stützt sich auf verschiedene Theorien aus dem Bereich CSCW und Management von Informationssystemen und untersucht den sozio-technischen Wandel durch die Implementierung und Nutzung von Kollaborationssystemen (Enterprise Collaboration Systems, ECS). Am Markt gibt es heute Standardsoftware für Unternehmenslösungen (z.B. HCL Connections), mit deren Hilfe man hochskalierende, integrierte Kollaborationsplattformen für den Digitalen Arbeitsplatz implementieren kann. Diese Kollaborationsplattformen bieten sowohl traditionelle Groupwarefunktionen (z. B. gemeinsame Arbeitsbereiche, synchronen und asynchronen Nachrichtenaustausch, Gruppenkalender, Dokumentbibliotheken) als auch Funktionalitäten sozialer Software (z. B. Wikis, Blogs, soziale Profile). Die Einführung von Kollaborationssystemen mit sozialen Funktionen verändert die Art und Weise, wie Einzelpersonen, Gruppen und Organisationen ihre Arbeit organisieren, verwalten und durchführen (Arbeitspraktiken), beispielsweise durch neue Formen der Teamzusammenarbeit, stärkeren Wissensaustausch, softwaregestützte Workflows oder veränderte Projektkoordination (Leonardi et al. 2013; Schubert und Williams 2013). Die Methoden, Veränderungsprozesse und langfristige Auswirkungen von ECS sind noch nicht vertieft erforscht und viele der laufenden Projekte haben nach wie vor einen Ausprobier- oder Experimentiercharakter.

IndustryConnect: Forschung mit Wirtschaftspartnern IndustryConnect begann im Jahre 2010 als eine Initiative des University Competence Center for Collaboration Technologies (UCT). Es ist ein Kooperationsprojekt zweier Lehrstühle am Fachbereich für Informatik einer deutschen Universität und einer Gruppe von Praktikern, die alle das gleiche, integrierte Kollaborationssystem (HCL Connections) im Einsatz haben. Die erste Version von Connections wurde von der Firma IBM entwickelt und kam 2007 auf den Markt. Die Praxisvertreter sind die Verantwortlichen für die Einführung der Kollaborationsplattform in ihren jeweiligen Organisationen. Die gemeinsamen Ziele dieses praxisorientierten Forschungsaustauschs sind 1) Forschung zu Problemen und Phänomenen rund um die Adaption von Kollaborationsplattformen, speziell die Kommunikation, Koordination und den aktiven Informationsaustausch unter den Mitarbeitenden, 2) Wissens- und Erfahrungsaustausch zwischen den Teilnehmern an der Initiative und 3) Entwicklung eines Verständnisses über den komplexen, sozio-technischen Wandel, den die Einführung von Kollaborationsplattformen und dem daraus entstehenden Digitalen Arbeitsplatz mit sich bringt.

IndustryConnect entspricht dem Multi-Client-Archetyp in der Klassifikation nach Schubert et al. (2015). Die Initiative bringt eine große Zahl von Unternehmen in 
einem 1:m-Setup mit einer Universität zusammen. Die Praxispartner haben ein gemeinsames Interesse an einem ganz spezifischen Thema (die Einführung von Kollaborationssystemen), das branchenunabhängig ist. Da es sich bei Kollaborationsprozessen (gemeinsame Arbeit) nicht um einen primären Geschäftsprozess handelt, spielen ggf. vorhandene Wettbewerbsbeziehungen zwischen den Unternehmen eine untergeordnete Rolle.

Die teilnehmenden Praktiker begrüßen den Informations- und Erfahrungsaustausch mit ihren Pendants in anderen Unternehmen und verpflichten sich mit ihrer Mitgliedschaft zu einer koordinierten, kooperativen Forschungstätigkeit. Sie haben ein Interesse daran, Geschichten aus der Praxis zu hören über ,Dinge, die für andere funktioniert haben“, d.h. Strategien, Methoden, Maßnahmen, Werkzeuge und Prozesse für die Einführung und erfolgreiche organisatorische Nutzung von Kollaborationssoftware. Die universitären Forscher sind daran interessiert, Einblicke in die gelebten Erfahrungen der aktuellen Praxis zu gewinnen, empirische Daten in den Unternehmen zu sammeln und diese Daten zu analysieren, um das sich noch in Entwicklung befindliche Forschungsthema zu verstehen und auf diese Weise geeignete Fragestellungen für die Theoriebildung zu identifizieren.

Die Mitgliedschaft in der Community wird auf zwei Arten gezielt verwaltet. Zunächst stammen alle Praxisvertreter aus Anwenderunternehmen (also Endanwender der Kollaborationssoftware). Softwareanbieter und IT-Berater sind ausdrücklich ausgeschlossen. Die Teilnehmer haben häufig bekundet, dass sie die Gelegenheit schätzen, Probleme offen zu diskutieren und sich auszutauschen, ohne dass ihnen ein IT-Anbieter und Berater eine Lösung dafür verkaufen möchte. Zweitens erfolgt die Aufnahme in die Forschungsgemeinschaft nur ,auf Einladung“ und nach Absprache mit den bestehenden Teilnehmern. Diese Vereinbarung stellt sicher, dass die Teilnehmer ein gemeinsames Interesse haben und dass die Motivation darin besteht, „Probleme zu lösen und Wissen auszutauschen“ und nicht „Geschäfte zu machen“ (d.h. Kunden zu gewinnen). Die Mitgliederzahl ist seit dem Start des Projekts stetig gewachsen. Die Initiative startete 2015 am ersten Workshop mit 11 Personen aus 10 Organisationen. Vier Jahre später umfasste die Community 68 Praxisvertreter aus 38 Organisationen. Dazu kommen auf Seiten der Universität 2 Universitätsprofessorinnen, 9 wissenschaftliche Mitarbeiter und 9 studentische Hilfskräften hinzu.

Die beteiligten Organisationen repräsentieren eine Vielzahl von Branchen. Um ein homogenes Vorwissen der Teilnehmer sicherzustellen, müssen neu eintretende Mitglieder zunächst einen „Einführungsworkshop“ durchlaufen, in dem die inhaltlichen und methodischen Grundlagen/Ergebnisse der ersten vier Jahre vermittelt werden. Das stellt sicher, dass sich die Wissensentwicklung in der Gruppe kontinuierlich weiterentwickelt und nicht stagniert. Die Wirtschaftspartner übernehmen abwechselnd die Ausrichtung der Workshops und übernehmen an diesen Tagen die Kosten für die Ausrichtung des Meetings in den Räumlichkeiten ihres Unternehmens.

University Competence Center for Collaboration Technology (UCT) Das Engagement der Forschenden in IndustryConnect ist freiwillig und kostenfrei für die beteiligten Organisationen. IndustryConnect wäre nicht möglich ohne das University Competence Center for Collaboration Technology (UCT). Das UCT stellt die 


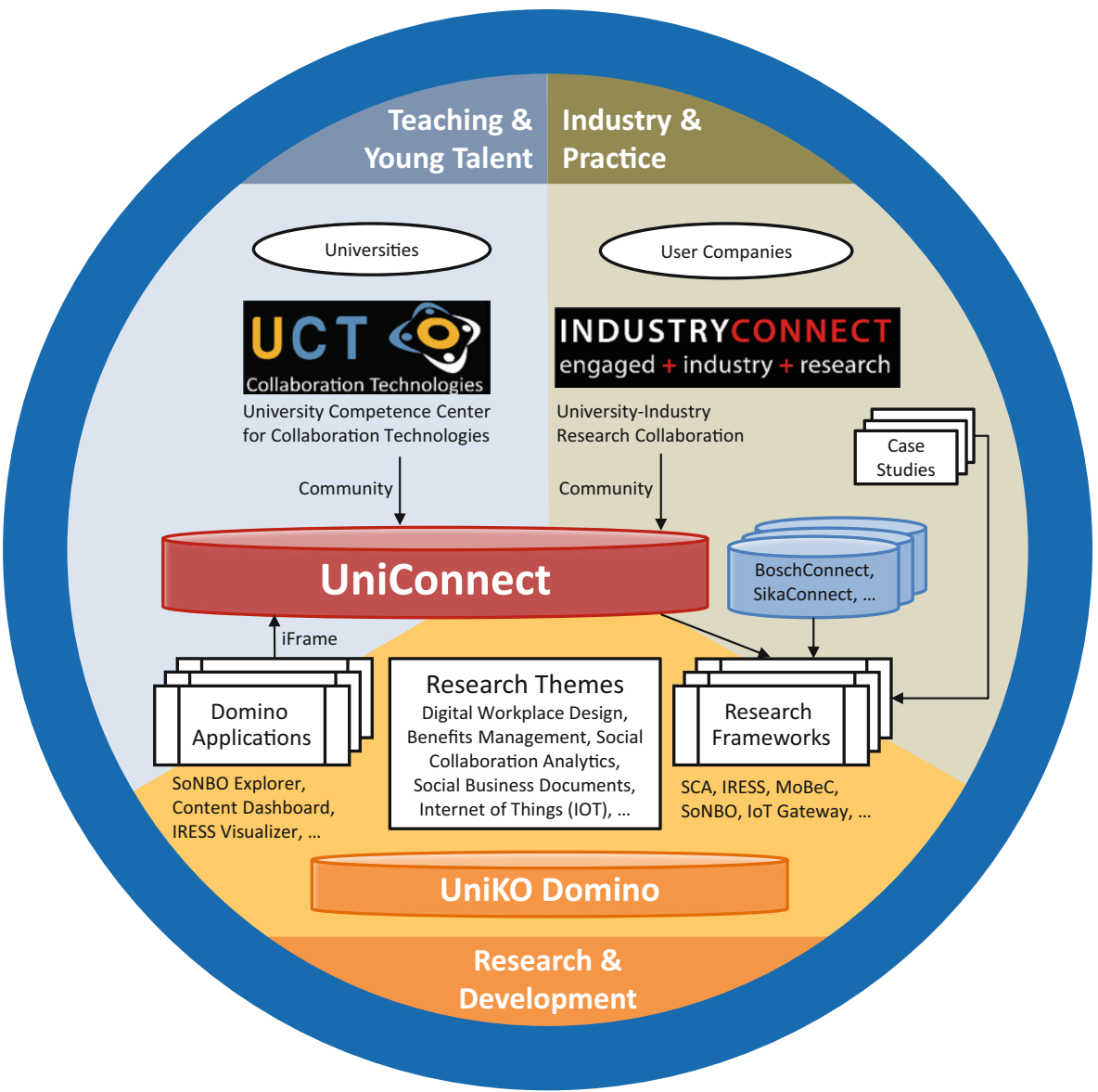

Abb. 1 Rahmenmodell praxisorientierter Forschung

Einbettung in den wissenschaftlichen Kontext sicher. Es ist auch die Hauptquelle der Finanzierung der wissenschaftlichen Mitarbeitenden (Drittmittel).

Der Kern des Kompetenzzentrums orientiert sich an Boyers (1990) vier ,Scholarships“, ein Begriff, der nicht direkt ins Deutsche übersetzt werden kann. Die vier Scholarships beschreiben den ,akademischen Leistungsauftrag“ und damit die „Handlungsgebiete von Hochschullehrern“ bzw. die Funktionen von Wissenschaft. Boyer argumentiert, dass ein guter Hochschullehrer eine integrierte Sicht auf die Bereiche Forschung und Lehre hat und eine Integration und Anwendung des Wissens vornimmt. Alle vier Handlungsgebiete fanden Einzug in das Rahmenmodell für die praxisorientierte Forschung, das in Abb. 1 dargestellt ist: Der linke Bereich fokussiert auf die Ausbildung junger Menschen (Scholarship of Teaching), was die Praxis heute ,young talent“" nennt. Der rechte Bereich zeigt die Gewinnung von Daten aus der Praxis, den Transfer der Ergebnisse und die anschließende Anwendung in Organisationen (Scholarship of Application). Der untere Bereich zeigt die Ergebnisse des klassischen Forschungsprozesses zum Zweck der Theoriebildung bzw. der 
Tab. 1 Bausteine des Rahmenmodells für praxisorientierte Forschung

\begin{tabular}{ll}
\hline $\begin{array}{l}\text { University Competence Center for Collaboration Technologies (UCT) } \\
\text { Aufgabe }\end{array}$ & Forschung, Bereitstellung von Lehrmaterial und Hosting von UniConnect \\
Charakter & Organisationale Einheit, die UniConnect zur Verfügung stellt \\
Plattform & $>1000$ Arbeitsbereiche (Communitys) \\
Mitglieder & $>30$ Universitäten in DACH \\
UniConnect & Integrierte Kollaborationsplattform basierend auf HCL Connections \\
Aufgabe & Herz und Motor von Lehre, Forschung und Entwicklung \\
Charakter & Akademische Einrichtungen (Universitäten) \\
Zielgruppe & SaaS, Hardware gehört der Universität Koblenz (UniKO) \\
Modus & \\
IndustryConnect & Gruppe führender Anwender integrierter Kollaborationsplattformen in DACH \\
Aufgabe & Kooperationsprojekt zwischen Wissenschaft und Wirtschaft \\
Charakter & Connections-Community auf UniConnect \\
Plattform & $>30$ Anwenderunternehmen, 1 Universität (Moderator) \\
Mitglieder & Physische (Plenums-)Treffen und bilaterale Forschungsprojekte \\
Modus &
\end{tabular}

Exploration durch prototypische Softwareentwicklung (Scholarship of Discovery). Das Rahmenmodell bringt Akteure verschiedener Disziplinen (wie z. B. Informatik, Management, Ingenieurwissenschaften, Soziologie) zusammen und sorgt damit für einen interdisziplinären Austausch (Scholarship of Integration).

Tab. 1 zeigt die wichtigsten „Bausteine“ des Rahmenmodells und beschreibt ihre Aufgabe, ihren Charakter und ihre Mitglieder. Das UCT repräsentiert die organisationale Einheit und damit die beteiligten Forschenden. UniConnect ist die zentrale (digitale) Kollaborationsplattform, auf der ein kontinuierlicher Austausch zwischen den Akteuren stattfindet. UniConnect hat mehr als 1000 Arbeitsbereiche (Communities), die sich in ihren Zwecken gemäß Boyer ebenfalls in Bereiche für Lehre, Forschung und Kooperationen mit der Praxis einteilen lassen. UniConnect ist das Herzstïck, der gemeinsame Arbeitsraum und Locus der Wissensbasis. Aufgrund der thematischen Ausrichtung auf den Digitalen Arbeitsplatz dient die Plattform auch gleichzeitig als Untersuchungsobjekt (z. B. für Social Collaboration Analytics).

\section{Faktoren für eine erfolgreiche praxisorientierte Forschung}

\subsection{Schaffung einer gemeinsamen Begriffswelt und Wissensbasis}

Schaffung einer gemeinsamen Begriffswelt Einer der wichtigsten Aspekte von multi-organisationaler, praxisorientierter Forschung ist das Schaffen eines gemeinsamen Begriffsverständnisses. Unternehmen haben idiosynkratische Sprachsysteme, so dass die fundamentalen Begriffe des Untersuchungsgebiets (Digitaler Arbeitsplatz) von Organisation zu Organisation unterschiedlich benannt sind. Während der Anfangszeit von IndustryConnect unterzogen die moderierenden Forscher die Gruppe einem Abstimmungs- und Einigungsprozess auf ein gemeinsames Vokabular. Ergeb- 
Tab. 2 Exemplarische Grundlagen für die Schaffung der gemeinsamen Begriffswelt

\begin{tabular}{ll}
\hline Zweck & Publiziert in \\
\hline $8 C$-Modell für Enterprise Information Management & \\
Überblick über die klassischen funktionalen Bereiche von CSCW (Communication, & (Williams 2011) \\
Cooperation, Content Combination, Coordination) und die damit verbundenen & \\
Managementaufgaben (Change, Contribution, Compliance, Content Management). & \\
Sinngebender Rahmen in der Form einer Klassifikation für die Diskussion über die & \\
Anwendungsbereiche & \\
IRESS-Rahmenwerk & \\
5-Ebenen-Modell für die Identifikation von Anforderungen an Kollaborations- & (Schubert und \\
software (IRESS = Identification of Requirements for Enterprise Social Software). & Glitsch 2016) \\
Etabliert grundsätzliche Konzepte für die Analyse des Einsatzes von Software am & (Glitsch und \\
Arbeitsplatz: 1) Use Cases (grober Einsatzbereich), 2) Collaboration Scenarios & Schubert 2017) \\
(systemgestützte Funktionsabläufe), 3) Social Documents and Actions (Ebene für & \\
Social Collaboration Analytics), 4) Software Features (Funktionalitäten in der Soft- & \\
ware) 5) Software Modules (Typen von Kollaborationssoftware) & \\
SCA-Framework & \\
Rahmenwerk für Social Collaboration Analytics (SCA). Beschreibt die möglichen & (Schwade und \\
$\begin{array}{l}\text { Datentypen, analysierbare Definitionen und zeigt die systematischen Schritte für } \\
\text { die Formulierung der tatsächlichen Abfragen }\end{array}$ & $\begin{array}{l}\text { Schubert 2017) } \\
\text { (Schwade und }\end{array}$ \\
& $\begin{array}{l}\text { Schubert 2018) } \\
\text { (Schubert et al. }\end{array}$ \\
& 2020) \\
\hline
\end{tabular}

nis dieses Prozesses war die Vereinbarung zur Nutzung von vorgegebenen Rahmenwerken, Definitionen und einer Ontologie für soziale Dokumente. Ausgangspunkt waren dabei jeweils die Theorien aus der zugrundeliegenden Domäne, in diesem Fall Computer Supported Cooperative Work $(C S C W)$. Darüber hinaus wurden die Teilnehmenden auch gezielt für die eingesetzten wissenschaftlichen Methoden und den Anspruch auf Rigorosität der Forschung sensibilisiert. Es wurde sichergestellt, dass alle Beteiligten sich, wie von Orme (2000) gefordert, in einer partnerschaftlichen Beziehung über das Forschungsdesign und die gewählten Methoden für die systematische Generierung von Wissen bewusst sind.

Für die Forschung im Bereich Digitaler Arbeitsplatz kommen die folgenden Rahmenwerke und die damit verbundenen Definitionen zum Einsatz (Tab. 2).

Gemeinsame Wissensbasis Eine entscheidende Erfolgskomponente ist die gemeinsame Wissensbasis, die durch die Aktivitäten in IndustryConnect über die Zeit entsteht. Jegliche Form der gemeinsamen Aktivität (Präsentationen und Diskussionen während Workshops, Fokusgruppen, Kreativitätstechniken, Fragebögen, etc.) wird laufend und systematisch von den Forschenden aufgezeichnet. Die Aufzeichnungen, die nach den physischen Treffen vorgenommen werden, sind für die Forschenden die Rohdaten für ihre weitere Forschung. Die Ergebnisse der Datenanalyse werden jeweils am nächsten Workshop an die Beteiligten zurückgespielt und in der Feedbackrunde damit auch in erster Runde validiert. Die Sammlung aller Aufzeichnungen und Auswertungen in der Form von Dokumenten, Tabellen, Infographs, Methodenbeschreibungen, Techniken und Guidelines, angereichert durch die direkt zu den Artefakten stattfindende Diskussion in der Community auf UniConnect (dem digitalen Arbeitsbereich der Gruppe), bildet die gemeinsame Wissensbasis. 


\subsection{Methoden und exemplarische Ergebnisse}

Aktionsforschung Die Arbeit in IndustryConnect orientiert sich an einer schrittweisen, sich zyklisch wiederholenden Aktionsforschung, die aus Planung, Implementation, Beobachtung, Reflexion, Theoretisierung und Handlung besteht (Baskerville und Wood-Harper 1996; Fals Borda 2001; Avison et al. 2018). Das Forschungsdesign kann nicht immer vollkommen im Vorhinein festgelegt werden (Patton 2002). Die sich ständig verändernde Natur des Digitalen Arbeitsplatzes erfordert flexible, interaktive Methoden der Primärdatenerhebung (Feldforschung), bei der Daten erhoben werden, ohne die genaue Fragestellung bereits zu kennen; denn oft ergeben sich die interessanten Fragen erst im Laufe der Erhebung. Um dem Anspruch einer rigorosen Forschung trotzdem entsprechen zu können, müssen die Erhebungsmethoden und die analytischen Rahmenwerke, die zur Identifikation von Schlüsselakteuren und Arbeitspraktiken in Kollaborationssystemen dienen, klar, robust und transparent an alle Forschungsbeteiligten kommuniziert werden. Um rigorose Forschungsergebnisse zu erzielen, muss eine deutliche Betonung auf Forschungsethik (Einverständniserklärung, engl.: informed consent) und Forschungsqualität vor allem in Bezug auf das Erzeugen zuverlässiger Evidenz gelegt werden. Die Forschung in IndustryConnect kommt dem nach, indem mindestens zwei Forschende bei allen Interviews anwesend sind und das Gegenlesen des Gesagten (bzw. der Interpretation davon) durch die Quellperson obligatorisch ist (engl.: member checking). Zusätzlich werden die Erkenntnisse kontinuierlich von der gesamten Gruppe diskutiert und damit überprüft. So wird der ,,interpretativen Natur der Daten“ (Baskerville und WoodHarper 1996) begegnet. Der bekannten Kritik an Einzelfallstudien (Flyvbjerg 2006), die sich nicht für Generalisierung eignet, kommen die Forschenden durch Datenerhebung in multiplen Organisationen nach (multiple case) (Thomas 2011). Dadurch kann geprüft werden, ob dieselbe Maßnahme in verschiedenen Organisationen dieselbe (oder eine andere) Wirkung erzielt.

Workshops Die 1- bis 2-tägigen IndustryConnect-Workshops finden zweimal pro Jahr statt und dienen primär der (moderierten) Diskussion, der Präsentation von Ergebnissen und Fallstudien, der interaktiven Arbeit an Ideen und Konzepten (unterstützt mit Metaplan-Technik), sowie - wie oben angesprochen - der Bildung des gemeinsamen Vokabulars über das Forschungsgebiet. Das Konzept stellt sicher, dass alle Beteiligten einen Redeanteil haben.

Fokusgruppen Fokusgruppen kommen in IndustryConnect besonders zu Beginn eines Forschungsprozesses zum Einsatz, wenn die eigentliche Forschungsfrage noch nicht vollkommen feststeht (Morgan 2009). Dabei werden Teilnehmer nach bestimmten Kriterien selektiert und in Teilgruppen eingeteilt. Jede Gruppe erhält ein vorgegebenes Thema, das für alle von gemeinsamem Interesse ist (Krueger und Casey 2015). In dem Gespräch stellen die Teilnehmer Fragen und berichten sich gegenseitig über Erfahrungen. Dabei kommen verschiedene Moderationstechniken zum Einsatz, um die Teilnehmer zu Diskussionen zu animieren und die Generierung von Ideen zu fördern. Die Interaktionen der Fokusgruppe werden von den begleiten- 

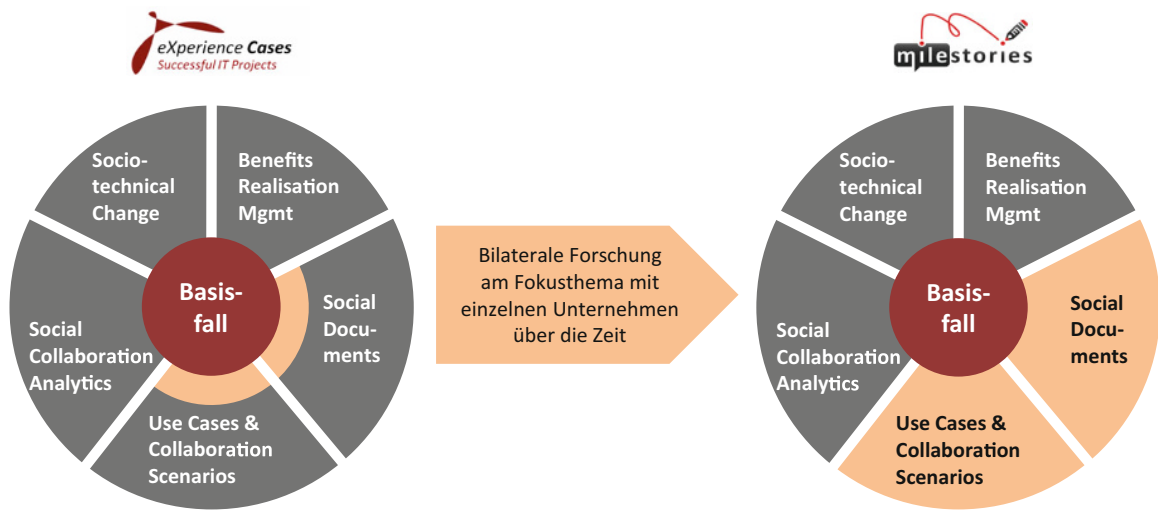

Abb. 2 Langzeitforschung: eXperience-Basisfall wird mit Milestories angereichert

den Forschern in Bild und Ton aufgezeichnet und für die anschließende Auswertung benutzt.

eXperience Fallstudien und Milestories eXperience-Fallstudien (Schubert und Wölfle 2007) und Milestories (Williams und Schubert 2017) sind die primären Methoden für die Erfassung und Dokumentation der (qualitativen) Unternehmensdaten (Abb. 2). Sie basieren auf Interviews mit Projektverantwortlichen (engl.: key informants). Während die Basisfallstudie eine Beschreibung eines IT-Einführungsprojekts und dessen verschiedene Aspekte (Geschäfts-, Prozess-, Applikations- und technische Sicht) enthält und einen Zeitpunktbericht darstellt, dienen Milestories zur Vertiefung einzelner Themen und speziell der Beobachtung von Veränderungen über die Zeit. Sie erlauben damit eine Zeitraumbetrachtung.

Der eXperience-Basisfall wird über die Zeit um Milestories ergänzt und damit zu einer Langzeitfallstudie entwickelt. Die Forschenden benutzen für die Erhebung einen einheitlichen, standardisierten Interviewleitfaden. Ein Forschender hat jeweils den Lead für einen Basisfall, ein zweiter Forschender begleitet das Interview und macht Notizen und hilft beim Schreiben der Fallstudie. Auf diese Weise stimmen sich mindestens zwei Personen über das Gehörte ab (inter author agreement). Jede Fallstudie wird von den Interviewten auf Vollständigkeit und Korrektheit überprüft (Smith 2002). Anschließend wird der Fall in einem zusätzlichen Qualitätsschritt von einer der verantwortlichen Professorinnen redaktionell überarbeitet, und es wird sichergestellt, dass alle relevanten Aussagen durch Verweise auf das Transkript gestützt werden. Im nächsten Schritt wird der Basisfall an einem uniinternen Workshop den Forschenden vorgestellt und diskutiert. Bei dieser Gelegenheit findet der angestrebte Wissenstransfer statt. Alle Basisfallstudien werden anschließend in der gemeinsamen Wissensbasis abgelegt und stehen allen Forschenden für ihre individuelle Forschung (speziell für Dissertationen) zur Verfügung.

Bei der eigentlichen Forschung kommt stets eine Kombination verschiedener Methoden zum Einsatz (Methodenmix). Die Ergebnisse sind unterschiedlicher Natur und reichen von reiner Wissensbildung (z.B. der Klassifikation von Phänomenen oder dem Einsatz von Analytics) über sinngebende Rahmenwerke bzw. Vorgehens- 
Tab. 3 Exemplarische Ergebnisse praxisorientierter Forschung

\begin{tabular}{|c|c|c|}
\hline Ergebnis & Methoden & Publikation \\
\hline $\begin{array}{l}\text { Digital Workplace Designs } \\
\text { Klassifikationsraster für typische Nutzungsmuster } \\
\text { bei der Nutzung von Kollaborationsplattformen } \\
\text { (6 Archetypen in } 2 \text { Dimensionen: people-focused/ } \\
\text { process-focused) }\end{array}$ & $\begin{array}{l}\text { Auswahl Basismodell (IRESS), } \\
\text { Use-Case-Analyse und themati- } \\
\text { sches Coding von } 13 \text { Fallstudien, } \\
\text { Grouping und Visualisierung der } \\
6 \text { Designs }\end{array}$ & $\begin{array}{l}\text { (Williams } \\
\text { und Schu- } \\
\text { bert 2018) }\end{array}$ \\
\hline $\begin{array}{l}\text { MoBeC } \\
\text { Rahmenwerk für die strukturierte Untersuchung } \\
\text { von Veränderungsprozessen bezüglich Motivation } \\
\text { und Nutzen von Kollaborationsplattformen (Moni- } \\
\text { toring Benefits Change). Modellelemente: Driver, } \\
\text { Output, Capability, Outcome, Benefit, Objective }\end{array}$ & $\begin{array}{l}\text { Kritischer Literaturreview, Aus- } \\
\text { wahl Basismodelle (OGC BRM } \\
\text { Framework und BND), } 3 \text { Tie- } \\
\text { fenfallstudien (Milestories), } \\
\text { Integration der Ergebnisse aus } \\
\text { Theorie und Praxis }\end{array}$ & $\begin{array}{l}\text { (Nitschke } \\
\text { und Wil- } \\
\text { liams } \\
\text { 2020) }\end{array}$ \\
\hline $\begin{array}{l}\text { Content Dashboard } \\
\text { Konzept für die Visualisierung von Social Docu- } \\
\text { ments in Graphenstruktur basierend auf einer selbst } \\
\text { entwickelten Ontologie (SocDOnt). Das Content } \\
\text { Dashboard ist eine Webapplikation für die real- } \\
\text { time Visualisierung von sozialen Dokumenten auf } \\
\text { UniConnect }\end{array}$ & $\begin{array}{l}\text { Design Science Research (DSR): } \\
\text { Fokusgruppe für die Bedarfser- } \\
\text { hebung, Entwicklung Ontologie } \\
\text { und Applikation, Evaluation der } \\
\text { Ergebnisse in Workshops mit der } \\
\text { Praxis }\end{array}$ & $\begin{array}{l}\text { (Mosen } \\
\text { et al. 2020) } \\
\text { (Williams } \\
\text { et al. 2020) }\end{array}$ \\
\hline
\end{tabular}

methoden bis hin zur Erstellung und Evaluation von Informatikartefakten wie Ontologien oder Prototypen.

Tab. 3 zeigt drei exemplarische Ergebnisse: 1) Theoriebildung (Digital Workplace Designs, ein empirischer Befund über den heutigen Einsatz von Kollaborationsplattformen), 2) Rahmenwerk/Methode zur Beschreibung und Visualisierung der Wirkungsmechanismen des sozio-technischen Wandels (MoBeC) und 3) Softwareapplikation für die Visualisierung von sozialen Dokumenten basierend auf einer im Rahmen von IndustryConnect entwickelten Ontologie (SocDOnt).

\section{Erfahrungen und Erkenntnisse}

Widmen wir uns nun der Frage, ob wir IndustryConnect auch aus heutiger Sicht noch einmal starten würden und ob wir evtl. heute etwas anders machen würden. Die Antwort ist ein klares Ja, wir würden es auf jeden Fall wieder tun, auch wenn es viele Situationen gab, in denen wir uns gefragt haben ,,was tun wir hier eigentlich?“ und ,ist das noch unser Auftrag als Universitätsprofessorinnen?“. Die Gewinnung von finanziellen Mitteln und Teilnehmern erfordert ein Maß an Kommunikation, das weit über das hinausgeht, was man aufgrund der Funktionsbeschreibung von einem Professor erwarten würde. Eine solche Initiative lohnt sich nach unserer Erfahrung vollumfänglich (highly rewarding), birgt aber auch Risiken. Die Abhängigkeit zwischen den drei Bausteinen 1) Einwerben von Drittmitteln (für die Stellen der wissenschaftlichen Mitarbeiter), 2) Betrieb der notwendigen Technologie (UniConnectPlattform) und 3) notwendigem Engagement der Beteiligten ist allgegenwärtig. Wie ein Jongleur muss man diese drei ständig im Auge behalten, Anträge schreiben, ein eigenes IT-Team aufbauen und ständige Beziehungspflege betreiben. Forschungsergebnisse müssen nicht nur in wissenschaftliche Publikationen umgesetzt werden, sondern zusätzlich in relevante Darstellungen für Praktiker umgeschrieben werden. 
Für diese „Extrameile“ müssen Doktoranden ein Verständnis aufbringen, denn diese Tätigkeit hilft ihnen nur mittelbar beim Erreichen ihres Ziels der Promotion. Hier kommt uns die besondere Situation in Deutschland entgegen, in der viele Doktoranden nach der Promotion in die Wirtschaft gehen (möchten). Für diese Zielgruppe bereitet die Arbeit in IndustryConnect einen fließenden Übergang und öffnet Türen.

Feedback von den beteiligten Wirtschaftsvertretern Die Rückmeldungen der Praxisvertreter in IndustryConnect waren für die Forscher bisher sehr motivierend. Es gab viele Beispiele und Belege dafür, dass die Forschungsergebnisse den Praktikern halfen, ihren eigenen organisatorischen Kontext zu verstehen, den sozio-technischen Wandel gezielt zu steuern und aus den Erfahrungen der anderen Praxisvertreter zu lernen. Durch Mund-zu-Mund-Propaganda wuchs die Gruppe laufend an. Über die Jahre hat sich eine Gemeinschaft gebildet, die sich gegenseitig unterstützt. So besteht heute auf Seiten der Praxisvertreter ein großes Verständnis für die Mechanismen der universitären Forschung, inklusive der Finanzierungsinstrumente, die IndustryConnect am Leben halten. So finden sich stets Freiwillige für das Ausstellen von Letters of Intent für spezielle Forschungsanträge (z. B. DFG, BMBF). Auch der Technologieprovider und Sponsor des UCT erkennt den Nutzen der gemeinsamen Forschung und verlängert daher seit nunmehr 10 Jahren den Kooperationsvertrag. Der wichtigste Ausdruck der Wertschätzung aber liegt in der ständigen Zurverfügungstellung von Zeit, in der Form von Interviews und dem Ausfüllen von Fragebögen. Die Teilnehmer sind sich bewusst, dass dies das „Eintrittsgeld“ ist, das sie für diese Community zahlen und sie melden zurück, dass sie dies interessant und motivierend finden.

Spezielles Doktorandenprogramm Wie zu Beginn des Beitrags angeführt, fordert das Hochschulgesetz von NRW explizit eine Doktorandenausbildung in Methoden und ethischen Grundsätzen. Die meisten Universitäten bieten spezielle inhäusige Doktorandenprogramme oder universitätsübergreifende Summer Schools an. Die sehr enge, positivistische Ausrichtung der Fähigkeiten junger Forschender und ihr ausschließlicher Fokus auf die Verstetigung (Tenure) und die dafür notwendigen Publikationen hat international Kritik an der Qualität von Doktorandenprogrammen aufkommen lassen (Davison und Martinsons 2011; Galliers 2011; Walsham 2012). Ein wesentlicher Aspekt des hier vorgestellten IndustryConnect-Rahmenmodells ist der Einbezug von Forschungstraining und der Betreuung von Nachwuchsforschenden in das Forschungsprogramm. Dies bedeutet, dass IndustryConnect auch Schulungen und moderierte Anwendung von Forschungsmethoden beinhaltet, einschließlich Entwicklung flexibler und interaktiver praxisorientierter Forschungsdesigns, Datensammlung mit mehreren Teilnehmern (z.B. Fokusgruppen, Delphi-Technik), gemeinsames Führen und Erstellen von Interviews, Forschungsethik, zentrales Forschungsdatenmanagement sowie eine Sensibilisierung für die Unterschiede in der Vermittlung von Ergebnissen an Wissenschaftler und Praktiker. IndustryConnect bietet den Doktoranden eine einzigartige Gelegenheit, eng mit Wirtschaftspartnern in einem kollaborativen Forschungsumfeld zusammenzuarbeiten. Dies setzt eine Bereitschaft voraus, kooperativ und offen zu sein und Erkenntnisse mit anderen im Team zu teilen. Durch die Mechanismen des Rahmenmodells unterziehen die 
Doktoranden sich und ihre Arbeit einer ständigen Überprüfung durch ein breites Publikum und müssen bereit sein, den erheblichen Aufwand für die Organisation und Verwaltung der verschiedenen Community-Aktivitäten zu betreiben. Sie sind an der Entwicklung der eXperience-Basisfälle und -Milestories und darüber hinaus speziell für ihre eigenen Themenbereiche verantwortlich. Jeder Kandidat betreut drei bis vier Unternehmen und stellt sicher, dass die Vorkehrungen für Besuche vor Ort geklärt sind, dass detaillierte Protokolle von Veranstaltungen und Sitzungen geführt und Zwischenergebnisse veröffentlicht werden. Die Teilnahme an praxisorientierter Forschung ist mit einem hohen Zusatzaufwand verbunden. IndustryConnect zieht daher eine spezielle Art von Doktoranden an, welche die Gelegenheit schätzen, als Teil einer Forschungsgemeinschaft zu arbeiten.

\section{Schlussbemerkungen}

Forschung mit Unternehmen und nicht über Unternehmen Der Nachweis des Werts der praxisorientierten Forschung und ihrer Ergebnisse erfordert besondere Aufmerksamkeit. Im Gegensatz zu der Art von Forschung über Unternehmen, bei der der Forschende lediglich Daten von Unternehmen sammelt, erfordert praxisorientierte Forschung einen signifikanten Vertrauens- und Beziehungsaufbau. Wir forschen nicht über Unternehmen, sondern mit Menschen in Unternehmen. Inwieweit sich die Teilnehmer als Teil der Community fühlen, zeigt sich in ihrer Bereitschaft, sich bei der Ausrichtung der Veranstaltung abzuwechseln und sich an den damit verbundenen administrativen Aufgaben zu beteiligen, etwa der Organisation der Räume und der Technik für den Workshop, Planung des abendlichen Social Events, der Organisation von Unterkünften, etc.

\section{Die letzte Meile gehen!}

Systems researchers who take their solutions through the last research mile may ultimately have the greatest impact on science and society (Nunamaker et al. 2015, S. 10).

Für uns als Forschende ist es sehr befriedigend, zu sehen, wie die von uns entwickelten Rahmenmodelle erfolgreich in Unternehmen angewendet werden, um Problembereiche und Handlungsfelder zu strukturieren. Wir dürfen miterleben, wie die selbsterstellen Prototypen im Unternehmenskontext funktionieren und dort Nutzen stiften. Der Mechanismus von IndustryConnect ist aus unserer Sicht ein schönes Beispiel, wie man Theorien und Methoden gemeinsam entwickeln und erfolgreich in die Praxis übertragen kann.

Was möchten wir weitergeben? Praxisorientierte Forschung ist kein leichter Weg und auch keiner, der gut in die Publish-or-Perish-Kultur passt. Junge Forschende haben keine Zeit, eine Forschungsinitiative aufzubauen, bevor sie ihr erstes Paper über die Auswertung der erhobenen Daten schreiben. Der Aufbau solcher Initiativen ist die Domäne ,älterer Kollegen“. Aber junge Forschende sind in einer glücklichen Situation, wenn sie sich in eine existierende Gruppe integrieren und an den bereits vorhandenen Daten partizipieren können. Die dargestellte Initiative gibt ihnen 
einen hervorragenden Zugang zu empirischen Daten und stellt auch sicher, dass die Forschungsfragen von hoher (wissenschaftlicher und praktischer) Relevanz sind.

Die Ausrichtung auf ein klares Fokusthema sowie der langfristige Aufbau einer vertrauensvollen Beziehung sind wesentliche Aspekte für die Gewinnung von Wirtschaftspartnern. Deutsche Universitäten haben ideale strukturelle Bedingungen für praxisorientierte Forschung. Die Organisation von Lehre und Forschung an Lehrstühlen ermöglicht den Aufbau von Forschungsgruppen, in denen mehrere Forschende unter der dauerhaft ausgelegten Leitung eines Professors langfristig an einem gemeinsamen Thema forschen. Das erlaubt das Verfolgen von Forschungsprogrammen statt einzelnen Forschungsprojekten.

Danksagung Ein großer Dank gilt dem Unternehmen IBM Deutschland, das 10 Jahre lang die Forschungsaktivitäten zum Thema Enterprise Collaboration Systems finanziell unterstützt hat und Hochschulen im DACH-Raum die Kollaborationsplattform UniConnect kostenfrei zur Nutzung zur Verfügung gestellt hat. Wir danken zudem den Praxisvertretern der Initiative IndustryConnect, die mit ihren offenen und konstruktiven Beiträgen die notwendigen (empirischen und systemtechnischen) Daten für die Langzeitforschung im Bereich Kollaborationssysteme zur Verfügung stellen.

Funding Open Access funding provided by Projekt DEAL.

Open Access Dieser Artikel wird unter der Creative Commons Namensnennung 4.0 International Lizenz veröffentlicht, welche die Nutzung, Vervielfältigung, Bearbeitung, Verbreitung und Wiedergabe in jeglichem Medium und Format erlaubt, sofern Sie den/die ursprünglichen Autor(en) und die Quelle ordnungsgemäß nennen, einen Link zur Creative Commons Lizenz beifügen und angeben, ob Änderungen vorgenommen wurden.

Die in diesem Artikel enthaltenen Bilder und sonstiges Drittmaterial unterliegen ebenfalls der genannten Creative Commons Lizenz, sofern sich aus der Abbildungslegende nichts anderes ergibt. Sofern das betreffende Material nicht unter der genannten Creative Commons Lizenz steht und die betreffende Handlung nicht nach gesetzlichen Vorschriften erlaubt ist, ist für die oben aufgeführten Weiterverwendungen des Materials die Einwilligung des jeweiligen Rechteinhabers einzuholen.

Weitere Details zur Lizenz entnehmen Sie bitte der Lizenzinformation auf http://creativecommons.org/ licenses/by/4.0/deed.de.

\section{Literatur}

Adler NJ, Hansen H (2012) Daring to care: scholarship that supports the courage of our convictions. J Manag Inq 21:128-139

Alvesson M, Sandberg J (2013) Constructing research questions. Doing interesting research. SAGE, London

Avison D, Malaurent J (2014) Is theory king? Questioning the theory fetish in information systems. J Inf Technol 29:327-336

Avison DE, Davison RM, Malaurent J (2018) Information systems action research: debunking myths and overcoming barriers. Inf Manag 55:177-187

Baskerville RL, Lyytinen K, Sambamurthy V, Straub D (2011) A response to the design-oriented information systems research memorandum. Eur J Inf Syst 20:11-15

Baskerville RL, Wood-Harper AT (1996) A critical perspective on action research as a method for information systems research. J Inf Technol 11:235-246

Boyer EL (1990) Scholarship reconsidered: the priorities of the professoriate. The Carnegie Foundation for the Advancement of Teaching, New York

Davison RM, Martinsons MG (2011) Methodological practice and policy for organisationally and socially relevant IS research: an inclusive-exclusive perspective. J Inf Technol 26:288-293

Dooley L, Kirk D (2007) University-industry collaboration. Eur J Innov Manag 10:316-332 
Fals Borda O (2001) Participatory (action) research in social theory: origins and challenges. In: Reason P, Bradbury $\mathrm{H}$ (Hrsg) Handbook of action research participative inquiry and practice. SAGE, London, S $27-37$

Flyvbjerg B (2006) Five misunderstandings about case-study research. Qual Inq 12:219-245

Galliers RD (2011) In celebration of diversity in information systems research. J Inf Technol 26:299-301

Galliers RD, Stein MK (2018) Information systems: to be, or not to be, a science? Is that the question? Commun Assoc Inf Syst 43:197-204

Glitsch JH, Schubert P (2017) IRESS: identification of requirements for enterprise social software. In: Procedia computer science. Elsevier, Barcelona, S 866-873

Krueger RA, Casey MA (2015) Focus groups: a practical guide for applied research, 5. Aufl. SAGE, Thousand Oaks

Leonardi PM, Huysman M, Steinfield C (2013) Enterprise social media: definition, history, and prospects for the study of social technologies in organizations. J Comput Commun 19:1-19

Lyytinen K, Baskerville R, Iivari J, Te'Eni D (2007) Why the old world cannot publish? Overcoming challenges in publishing high-impact IS research. Eur J Inf Syst 16:317-326

Morgan DL (2009) Focus groups. Annu Rev Sociol 22:129-152

Mosen J, Williams SP, Schubert P (2020) Visualizing social documents as traces of collaborative activity in enterprise collaboration platforms. 53rd Hawaii International Conference on System Sciences.

Nitschke C, Williams SP (2020) Monitoring and understanding enterprise collaboration platform outcomes and benefits change. 53rd Hawaii International Conference on System Sciences.

Nunamaker JF, Briggs RO, Derrick DC, Schwabe G (2015) The last research Mile: achieving both rigor and relevance in information systems research. J Manag Inf Syst 32:10-47

Orme J (2000) Interactive social sciences: patronage or partnership? Sci Public Policy 27:211-219

Österle H, Becker J, Frank U et al (2010) Memorandum zur gestaltungsorientierten Wirtschaftsinformatik. Schmalenbachs Z betriebswirtsch Forsch 62:664-672

Österle H, Becker J, Frank U et al (2011) Memorandum on design-oriented information systems research. Eur J Inf Syst 20:7-10

Patton MQ (2002) Qualitative research and evaluation methods, 3. Aufl. SAGE, Thousand Oaks

Schneiderman B (2016) The new ABCs of research: achieving breakthrough collaborations. Oxford University Press, Oxford

Schön DA (2001) The crisis of professional knowledge and the pursuit of an epistemology of practice. In: Raven J, Stephenson J (Hrsg) Competence in the learning society. Peter Lang, New York, S 185-207

Schubert P, Bjørn-Andersen N, Kilian T (2015) Archetypes for engaged scholarship in IS. Int J Inf Syst Manag 1:219-239

Schubert P, Glitsch JH (2016) Use cases and collaboration scenarios: how employees use socially-enabled enterprise collaboration systems (ECS). Int J Inf Syst Proj Manag 4:41-62

Schubert P, Mosen J, Schwade F (2020) Metrics for analyzing social documents to understand joint work. 53rd Hawaii International Conference on System Sciences.

Schubert P, Williams SP (2013) The concept of social business: oxymoron or sign of a changing work culture? 26th Bled eConference, Bled, Slovenia, S 222-235

Schubert P, Wölfle R (2007) The eXperience methodology for writing IS case studies. Americas Conference on Information Systems, S 1-15

Schwade F, Schubert P (2017) Social collaboration analytics for enterprise collaboration systems: providing business intelligence on collaboration activities. 50th Hawaii International Conference on System Sciences, S 401-410

Schwade F, Schubert P (2018) A survey on the status quo of social collaboration analytics in practice. European Conference on Information Systems.

Smith JA (2002) Evolving issues for qualitative psychology. In: Richardson JTE (Hrsg) Handbook of qualitative research methods for psychology and the social sciences. Blackwell, Malden, S 189-202

Thomas G (2011) A typology for the case study in social science following a review of definition, discourse, and structure. Qual Inq 17:511-521

Walsham G (2012) Are we making a better world with ICTs? Reflections on a future agenda for the IS field. J Inf Technol 27:87-93

Williams SP (2011) Das 8C-Modell für kollaborative Technologien. In: Schubert P, Koch M (Hrsg) Wettbewerbsfaktor Business Software. Hanser, München, S 11-21

Williams SP, Mosen J, Schubert P (2020) The structure of social documents. 53rd Hawaii International Conference on System Sciences.

Williams SP, Schubert P (2017) Connecting industry: building and sustaining a practice-based research community. 50th Hawaii International Conference on System Sciences, S 5400-5409 
Williams SP, Schubert P (2018) Designs for the digital Workplace. In: Procedia computer science. Elsevier, Amsterdam, S 478-485

Winter SJ, Butler BS (2011) Creating bigger problems: grand challenges as boundary objects and the legitimacy of the information systems field. J Inf Technol 26:99-108 